\title{
Comparative Study of Transvaginal Sonography and Hysteroscopy for Detection of Pathological Endometrial Lesions in Women with Perimenopausal Bleeding
}

\author{
Emad Abd El-Rahman El-Tamamy, Mofeed Fawzy Mohamed and Gamal Kamel El-Sayed * \\ Obstetrics and Gynecology Department, Faculty of Medicine, Al-Azhar University \\ *Corresponding Author: Gamal Kamel El-Sayed, Phone No.: (+2) 01064604025, E-mail: gamalkamel346@ gmail.com
}

\begin{abstract}
Background: abnormal uterine bleeding is a frequent condition in gynecology. It may impact physical, emotional sexual and professional aspects of the lives of women, impairing their quality of life. In cases of acute and severe bleeding, women may need urgent treatment with volumetric replacement and prescription of haemostatic substances. In some specific cases with more intense and prolonged bleeding surgical treatment may be necessary. Aim of the Work: this study aimed to estimate the diagnostic accuracy of two dimensional transvaginal ultrasound and hysteroscopy compared to the histopathology in evaluation of uterine cavity lesions in peri menopausal women with abnormal uterine bleeding. Patients and Methods: this was a prospective cohort study and it included 50 patients from Obstetrics and Gynecology Outpatient Clinic at Al-Hussein and Bab El-Shearia (Sayed Galal) Hospitals, Al-Azhar University during the period from October 2017 to May 2018. Results: 2D TVS were more sensitive and a little more accurate than hysteroscopy in detecting endometrial hyperplasia, while hysteroscopy showed higher specificity. Hysteroscopy showed highest accuracy for diagnosis of the endometrial polyp and was more sensitive, specific and accurate than 2D TVS in this diagnosis. 2D TVS were more sensitive and accurate than hysteroscopy in the diagnosis of adenomyosis, but both showed same specificity. For differentiating normal from abnormal endometrial cavity both 2D TVS and hysteroscopy showed high accuracy, but U/S was more sensitive and a little more accurate than hysteroscopy, while the last was more specific. Both 2D TVS and hysteroscopy ignore the diagnoses of one lesion when endometrial hyperplasia, endometrial polyp or adenomyosis were found in one case. Conclusion: endometrial hyperplasia was the commonest observed endometrial abnormality in our patient sample and the second most common pathology was the endometrial polyp.
\end{abstract}

Keywords: transvaginal sonography, perimenopausal bleeding, pathological endometrial lesions

\section{INTRODUCTION}

Abnormal uterine bleeding (AUB) is the main reason for women to go to gynecologists and accounts for two thirds of all hysterectomies ${ }^{(\mathbf{1})}$. AUB is responsible for as many as one-third of all outpatient gynecologic visits and this proportion rise to $69 \%$ in a the perimenopausal group ${ }^{(2)}$. In perimenopausal women, AUB is diagnosed when there is a substantial change in frequency, duration, or amount of bleeding during or between periods. In postmenopausal women, any vaginal bleeding 1 year after cessation of menses is considered abnormal and requires evaluation ${ }^{(3)}$.The cause of AUB in perimenopausal women was found in $50 \%$ to $60 \%$ of cases. The remaining cases, where no organic cause was found, were classified as DUB ${ }^{(\mathbf{1})}$.History focused on identifying the type of AUB as ovulatory, anovulatory or anatomic in order to guide treatment. Ovulatory bleeding is more common, usually cyclic and can be associated with midcycle pain, premenstrual symptoms and dysmenorrhea (4). Anovulatory bleeding occurs more frequently at the extremes of reproductive age and in obese women. It is usually irregular and often heavy. It poses a higher risk of endometrial hyperplasia ${ }^{(5)}$. Fibroids or polyps are the most common cause of anatomic AUB twenty to forty percent of women had fibroids. These women might present with abnormal bleeding, anemia, pain and occasionally infertility ${ }^{(6)}$.Physical examination included looking for evidence of systemic diseases. Pelvic and bimanual examinations were done to detect diseases in the genital tract. Cervical cytology analysis should be current and normal and cervical and vaginal swabs should be assessed to rule out infection ${ }^{(7)}$.Investigations can include pregnancy testing if indicated and a $\mathrm{CBC}$ with ferritin. A serum ferritin level may be useful if there is any doubt about iron deficiency, but should not be a routine investigation. Other investigations might be done on the basis of clinical suspicion 
${ }^{\text {(3) }}$.Diagnostic procedures for anatomic changes and for endometrial carcinoma included ultrasonography, diagnostic hysteroscopy, sonohysterogram, and dilation and curettage $(D \& C)^{(7)}$. Ultrasonography is used to look for ovarian or uterine disease and endometrial biopsy (EMB). Transvaginal Sonography (TVS) was $80 \%$ sensitive and $69 \%$ specific for fibroids and polyps and was superior to transabdominal ultrasound (TAS). If possible, TVS should be performed on days 4 to 6 of the menstrual cycle. In perimenopausal women, there is no known correlation between endometrial thickness seen on ultrasound scan and endometrial disease ${ }^{(8)}$. EMB is a simple office procedure that can be done by physicians. The age-standardized incidence rate of endometrial cancer was 18 per 100,000 in US whites. One in 4 cases of endometrial carcinoma occurred before menopause, so biopsy should be considered for high risk perimenopausal patients. In Egypt the agestandardized incidence rates of endometrial cancer was 3.5. Per 100,000, but age-specific incidence rates were 10.9 per 100,000 for those women aged between 50-59 yr. The percentage of cases below the age of 50 years for uterine cancer was highest in the Egyptians (33.1\%) while, for US SEER it was $15.5 \%{ }^{(9)}$. EMB had high accuracy in diagnosing endometrial cancer and hyperplasia and should be employed when serious endometrial disease was suspected in both pre- and postmenopausal women ${ }^{(\mathbf{1 0})}$.Even in the presence of fibroids. EMB produced an adequate sample more than $85 \%$ of the time and detects $87 \%$ to $96 \%$ of endometrial carcinoma (8). D\&C does not sample the entire endometrium and can miss up to $10 \%$ of disease. Due to its associated operative risks, D\&C was falling out of favors ${ }^{(11)}$.Hysteroscopy allows direct visualization of the endometrial cavity and is usually combined with EMB. Saline sonohysterogram involves introducing 5 to $15 \mathrm{ml}$ of saline solution into the uterine cavity followed by a TVS scan that might help diagnose an intrauterine mass ${ }^{(8)}$.In perimenopausal women with no obvious cause of AUB, the need for further investigations is controversial. If women have only normal variations in their menstrual cycles, reassurance is all that is required. For instance, following times of stress, abnormal menstrual cycles often revert to normal after a few months. If physicians are concerned about fibroids or polyps, endometrial hyperplasia, or carcinoma, further workup and treatment are important ${ }^{(7)}$.
Evaluation of AUB in perimenopausal women is challenging. As a result of the decline in ovarian function, changes in menstrual cycles are common in these women ${ }^{(\mathbf{1 2})}$. As with postmenopausal bleeding, abnormal perimenopausal bleeding is associated with endometrial carcinoma in approximately $10 \%$ of cases so evaluation of women's risk factors for endometrial hyperplasia or carcinoma is recommended ${ }^{(13)}$. According to guidelines Vilos et al. ${ }^{\left({ }^{(8)}\right.}$ all perimenopausal women with irregular bleeding warrant further investigation. It is important to note, however, that the highest risk of endometrial carcinoma is among women who weigh $\geq 90 \mathrm{~kg}$ and who are 45 years or older ${ }^{(\mathbf{1 3})}$.

\section{AIM of the WORK}

The aim of this study was to estimate the diagnostic accuracy of two dimensional transvaginal ultrasound and hysteroscopy compared with histopathology in evaluation of uterine cavity lesions in perimenopausal women with abnormal uterine bleeding.

\section{PATIENTS and METHODS}

This prospective cohort study included 50 patients and they were included from those attending the Obstetrics and Gynecology Outpatient Clinic at Al-Hussein and Bab ElShearia (Sayed Galal) Hospitals, Al-Azhar University during the period from October 2017 to May 2018.

\section{Ethical committee:}

This study designed according to ethical committee rules of obstetric and gynecology, for all women in the study explanation of the study procedures was done and informed consent was taken.

\section{Inculsion Criteria}

The patients should have the following criteria:

1- Perimenopausal age group (40-55 years).

2- Having abnormal uterine bleeding due to endometrial or uterine cavity lesions as diagnosed by TVS.

3- No evident drug intake that can lead to vaginal bleeding.

4- No evident general cause that can cause vaginal bleeding.

5- No vaginal, vulval or cervical causes of bleeding.

6- Preliminary ultrasound to exclude adnexal masses.

7- Not using any local or hormonal method of contraception.

\section{Exclusion Criteria}

The following patients will be excluded from the current study: 
1- Not having abnormal uterine bleeding due to endometrial or uterine cavity lesions as diagnosed by TVS.

2- Evident drug intake that can lead to vaginal bleeding.

3- Evident general cause that can cause vaginal bleeding.

4- Vaginal, vulval or cervical causes of bleeding.

5- Using any local or hormonal method of contraception.

Methods

All the patients were admitted to AlHussein and Bab El-Shearia (Sayed Galal) Hospitals, Al-Azhar University; they were not categorized into groups; all the patients were subjected to the following points:

1. Detailed clinical history: included the following points:

Present history of bleeding including onset, course, duration, and criteria of bleeding pattern.

Menstrual history, history of any drug intake, blood disease or any medical disorders were considered. transfusion.

Past history of operations or blood

2. Clinical examination was done including general, abdominal and Pelvic examination in a form of bimanual and speculum examination to detect any abnormal findings and to exclude any local cause of bleeding.

3. Laboratory investigations including CBC, coagulation profile, fasting and post prandial blood sugar, liver and kidney function and pregnancy test.

4. Conventional 2D- Transvaginal ultrasonography measuring uterine size and endometrial thickness, looking for endometrial polypi and any relevant adnexal pathology.

5. Hysteroscopic examination: under general anesthesia looking for gross endometrial lesions, polyps, and abnormal vascular patterns.

6. Fractional curettage to all the patients, and in some of them hysterectomy were done later. All the specimens were placed in formalin $10 \%$ and sent for histopathological correlation.

7. Histopathological examinations of endometrial tissue using fractional curettage or hysterectomy specimen's when available.

\section{Ultrasonography}

With an empty bladder, the patient was examined in the lithotomy position using a machine with a transvaginal probe (GE 6.5 MTZ). The probe was covered with a glove and small amount of gel was applied to the probe before insertion into the vagina.To obtain images in varying directions, planes and depths the probe should move slowly, in various planes(in anteroposterior plane rocking movement, in the horizontal plane-panning movement-) and at various depth by pushing and pulling the probe along the vagina.

\section{Hysteroscopy}

Diagnostic hysteroscopy was carried out to all patients under general anesthesia by an operator who was blinded to the ultrasound findings. it was a rigid continuous flow panoramic hysteroscopy by $25 \mathrm{~cm}$ in length, 5 $\mathrm{mm}$ diameter of an outer sheath and $30^{\circ}$ fibroptic lens. The technique used to provide constant uterine distention was by attaching plastic bags of saline or glycin solution. Infusion pressure was elevated by pneumatic cuff under manometric control at a pressure of 100-120 mm HG.The procedure was monitored using a single chip video and the image was displayed on a monitor visible to the operator. Hysteroscopic picture which appeared on the monitor is transmitted through the optic from the camera which was fitted on the eye piece where the panoramic diagnostic hysteroscopy could be performed with better visualization and accuracy.

\section{Steps of the Procedure:}

1. The patient is put in the lithotomy position.

2. Complete aseptic technique by cleaning the area around the vulva, vagina and the cervix with a non foaming aseptic solution.

3. Emptying the bladder by a metal catheter.

4. Examination under anesthesia.

5. Introduction of a vaginal retractor into the vagina to expose the cervix and a single toothed volsellum is applied to the anterior lip of the cervix.

6. Dilatation of the cervix may be needed up to Hegar no. 6 but it is better to be avoided as the tight cervical os avoids loss of the distending medium.

7. The hysteroscope is introduced through the external cervical os under direct vision or with the application of the camera before introduction 
8. Once the cavity was entered, a panoramic view of the uterine cavity is seen to exclude uterine malformation or deformed cavity.

9. Once the telescope was introduced in the cavity, examination was done systematically: first the fundus, then anterior, then posterior then lateral walls of the uterus ending by visualization of the uterotubal junctions.

10. The thickness, the color, the vasculature and the consistency of the mucous membrane lining the uterine cavity was observed and recorded the aspect of the endocervical mucosa and the endometrium should be described.

11. If there is any intrauterine pathology detected, the shape, the size and the site is estimated. If submucosal fibroid was found, resectoscope was used to remove it. If endometrial hyperplasia was suspected, a groove was always done with the optic by passing it through the posterior uterine wall and the thickness was estimated.

12. At the end of the procedure, the hysteroscope was slowly withdrawn through the cervical canal which was visualized for staging in cases of endometrial carcinoma.

\section{Fractional curettage}

The first sample was taken from the endocervical canal before cervical dilatation, then cervical dilatation up to 7-8 Hegar. Endometrial polyp removal using ring forceps was carried out following hysteroscopic localization of these polypi. A sharp curette was introduced and curettage starting first with the fundus then posterior wall then anterior wall then right then left lateral walls. The sample is placed in formalin $10 \%$ until histological staining with haematoxylin and eosin for histopathological examinations by the pathologist.

\section{Statistical Methods}

Data was statistically represented by the term of range, mean, standard deviation (+SD) and percentages. Accuracy was represented using the terms of sensitivity, specificity, positive predictive value, negative predictive value and overall accuracy. All statistical calculations were done using computer programs Microsoft Excel version 7 (Microsoft Corporation NY, USA).
To analyze the diagnostic accuracy of hysteroscopy and 2D-TVS specifically for intrauterine disorders ( $\mathrm{X}$ diagnosis), such as endometrial polyps and hyperplasia $2 \times 2$ tables were constructed.

\begin{tabular}{|l|l|l|}
\hline \multirow{2}{*}{$\begin{array}{l}\text { Findings by TVS } \\
\text { and hysteroscopy }\end{array}$} & \multicolumn{2}{|l|}{ Histopathology findings } \\
\cline { 2 - 3 } X diagnosis & $\begin{array}{l}\text { other } \\
\text { diagnosis }\end{array}$ \\
\hline X diagnosis & $\begin{array}{l}\text { Truly } \\
\text { identified (a) }\end{array}$ & $\begin{array}{l}\text { Falsely } \\
\text { identified (b) }\end{array}$ \\
\hline Other diagnosis & $\begin{array}{l}\text { Falsely } \\
\text { excluded (c) }\end{array}$ & $\begin{array}{l}\text { Truly } \\
\text { excluded (d) }\end{array}$ \\
\hline
\end{tabular}

To analyze the diagnostic accuracy for the diagnoses of all lesions, two outcomes were considered: intracavitary abnormality and normal uterine cavity Data were abstracted as 2 X 2 tables of hysteroscopy or US result (positive or negative for intrauterine abnormality) and the histological results (normal or abnormal).

To calculate sensitivity, specificity, positive predictive value, negative predictive value and overall accuracy following equations were used.

- Sensitivity $=a / a+c$

- Specificity $=\mathrm{d} / \mathrm{b}+\mathrm{d}$

- Positive predictive value $=\mathrm{a} / \mathrm{a}+\mathrm{b}$

- Negative predictive value $=\mathrm{d} / \mathrm{c}+\mathrm{d}$

- Overall accuracy $=a+d / a+b+c+d$.

RESULTS

All the patients were complained of AUB $(100 \%)$. AUB was associated with pain in $38 \%$ of the cases and with infertility in $10 \%$ of the cases.

Table 1: clinical presentation of the patient's sample

\begin{tabular}{|l|l|l|}
\hline Presentation & Number & percent \\
\hline Abnormal uterine bleeding & 50 & $100 \%$ \\
\hline$>$ Menorrhagia & 20 & $40 \%$ \\
\hline$>$ Menometrorrhagia & 17 & $34 \%$ \\
\hline$>$ Metrorrhagia & 13 & $26 \%$ \\
\hline PAIN & 19 & $38 \%$ \\
\hline$>$ Vague pelvic pain & 9 & $18 \%$ \\
\hline$>$ Dysmenorrhea & 8 & $16 \%$ \\
\hline$>$ Dyspareunia & 2 & $4 \%$ \\
\hline INFERTILITY & 5 & $10 \%$ \\
\hline
\end{tabular}

Table 2: accuracy measures of 2D- TVS for diagnosing some uterine lesions

\begin{tabular}{|l|l|l|l|}
\hline Lesion & $\begin{array}{l}\text { Endometri } \\
\text { al } \\
\text { Hyperplas } \\
\text { ia }\end{array}$ & $\begin{array}{l}\text { Endometri } \\
\text { al Polyp }\end{array}$ & $\begin{array}{l}\text { Adenomyo } \\
\text { sis }\end{array}$ \\
\hline $\begin{array}{l}\text { Sensitivi } \\
\text { ty }\end{array}$ & $60 \%$ & $76.92 \%$ & $68.18 \%$ \\
\hline $\begin{array}{l}\text { Specifici } \\
\text { ty }\end{array}$ & $84 \%$ & $91.89 \%$ & $98.78 \%$ \\
\hline PPV & $78.94 \%$ & $76.92 \%$ & $93.75 \%$ \\
\hline NPV & $67.74 \%$ & $91.89 \%$ & $92.04 \%$ \\
\hline
\end{tabular}




\begin{tabular}{|l|l|l|l|}
\hline $\begin{array}{l}\text { Accurac } \\
\mathbf{y}\end{array}$ & $72 \%$ & $88 \%$ & $94 \%$ \\
\hline
\end{tabular}

2D ultrasound showed good sensitivity in detection of endometrial polyp, highest specificity and accuracy was for adenomyosis.

Table 3: accuracy measures of hysteroscopy for diagnoses of some uterine lesions

\begin{tabular}{|l|l|l|l|}
\hline Lesion & $\begin{array}{l}\text { Endometri } \\
\text { al } \\
\text { Hyperplas } \\
\text { ia }\end{array}$ & $\begin{array}{l}\text { Endometri } \\
\text { al Polyp }\end{array}$ & $\begin{array}{l}\text { Adenomyo } \\
\text { sis }\end{array}$ \\
\hline $\begin{array}{l}\text { Sensitivi } \\
\text { ty }\end{array}$ & $40.38 \%$ & $92.3 \%$ & $40.9 \%$ \\
\hline $\begin{array}{l}\text { Specifici } \\
\text { ty }\end{array}$ & $98.07 \%$ & $94.59 \%$ & $98.78 \%$ \\
\hline PPV & 95.45 & $85.71 \%$ & $90 \%$ \\
\hline NPV & 62.19 & $97.22 \%$ & $86.17 \%$ \\
\hline $\begin{array}{l}\text { Accurac } \\
\text { y }\end{array}$ & $70 \%$ & $94 \%$ & $88 \%$ \\
\hline
\end{tabular}

Hysteroscopy was poorly sensitive, but highly specific for both endometrial hyperplasia and adenomyosis. For endometrial polyp hysteroscopy was highly sensitive, specific and accurate.

Table 4: accuracy measures of hysteroscopy and 2D TVS for the diagnoses of all lesions

\begin{tabular}{|l|l|l|}
\hline $\begin{array}{l}\text { Diagnosis } \\
\text { Tool measure }\end{array}$ & TVS & Hysteroscopy \\
\hline Sensitivity & $92.3 \%$ & $78.75 \%$ \\
\hline Specificity & $72.72 \%$ & $95.83 \%$ \\
\hline PPV & $92.3 \%$ & $98.43 \%$ \\
\hline NPV & $72.72 \%$ & $57.5 \%$ \\
\hline Accuracy & $88 \%$ & $84 \%$ \\
\hline
\end{tabular}

Ultrasound was more sensitive and more accurate than hysteroscopy for detection of uterine lesions but hysteroscopy show higher specificity.

\section{DISCUSSION}

Abnormal uterine bleeding is a common presentation in primary care, but is often complex and difficult to diagnose. One study found that menstrual disorders were the reason for $19.1 \%$ of 20.1 million visits to clinicians for gynecologic conditions over a 2year period in USA. Up to $33 \%$ of women referred to gynecological outpatient clinics had AUB and this proportion rises to $69 \%$ in a perimenopausal group. Furthermore, a reported $25 \%$ of gynecologic surgeries involved AUB. Between 30 to $50 \%$ of all hysterectomies are due to abnormal bleeding and fibroids, yet 20 $\%$ of hysterectomies are associated with normal uterine pathology ${ }^{(14)}$. The causes of AUB in perimenopausal women can be variable, from simple DUB without any organic cause to the endometrial cancer. Because the risk of developing endometrial cancer increases with age and in women aged 40 to 49 years, the incidence of endometrial carcinoma is 36.5 cases per 100,000 and knowing that more than $90 \%$ of patients with endometrial cancer will present with abnormal vaginal bleeding, whether it is menorrhagia, metrorrhagia, these symptoms should be thoroughly evaluated. For the evaluation of this condition; invasive procedures such as hysteroscopy and curettage are commonly used ${ }^{(\mathbf{1 5})}$. In our study 50 patients complained of perimenopausal bleeding were included and they were attending the Obstetrics and Gynecology Outpatient Clinic at AlHussein and Bab El-Shearia (Sayed Galal) Hospitals, Al-Azhar University. All women were subjected to conventional TVS measuring the endometrial thickness, detailed hysteroscopy and endometrial curettage for histopathologic examination. In our study histopathology findings revealed single lesion in 25 cases $(50 \%)$, two lesions in 14 cases (28\%) and normally functioning endometrium in 11 cases (22\%).Endometrial hyperplasia, which was the commonest finding by histopathology, was found in 25 patients (50\%) was accompanied by endometrial polyp in 7 cases (14\%) and by adenomyosis in 5 cases (10\%).Histopathology diagnosed endometrial polyp in 13 cases (26\%), adenomyosis in 10 cases $(20 \%)$ and submucosal fibroid was found in only 2 cases $(4 \%)$. endometritis, atrophic endometrium and adenomyomatous polyp each were found in one case (2\%) No endometrial cancer was diagnosed in this cohort of women.By 2D ultrasound normal uteri was found in 11 cases (22\%), endometrial hyperplasia was detected in 19 cases $(38 \%)$ and in 3 of them it was accompanied by adenomyosis. Endometrial polyp was detected in 13 cases (26\%), adenomyosis in 7 cases (14\%) and submucosal fibroid was detected in 3 cases (6\%).Using hysteroscopy commonest findings was normal uterine cavity which was detected in 19 cases (38\%) followed by endometrial polyp in 14 cases (28\%), endometrial hyperplasia in 10 cases $(20 \%)$, adenomyosis in 4 cases (8\%) and finally submucosal fibroid was found in 3 cases (6\%).In our study when we compared 2D ultrasound and hysteroscopy findings with the 
histopathologic findings of tissue obtained by hysterectomy or D\&C, we found that:

$>$ For diagnosing endometrial hyperplasia 2D TVS we detected $60 \%$ of pathologically diagnosed cases (sensitivity), could exclude $84 \%$ of pathologically excluded cases (specificity). From those testing positive by ultrasound $78.94 \%$ was truly positive (PPV) and from those testing negative $67.74 \%$ was truly negative (NPV). 2D TVS was accurate in of $72 \%$ of cases.2D TVS misdiagnosed 2 cases of endometrial hyperplasia as polyp and missed one. When endometrial hyperplasia was accompanied by polyp (7 cases),2D TVS diagnosis was hyperplasia (5 cases) and polyp (2 cases) but did not diagnosed both in one case and when endometrial hyperplasia was accompanied by adenomyosis ( 5 cases) 2D TVS diagnosis was hyperplasia in 1 case, adenomyosis in 1 case and both in 3 cases.

Hysteroscopy showed sensitivity $40.38 \%$, specificity $98.07 \%$, PPV 95.45, NPV 62.19 and accuracy $70 \%$ in detecting endometrial hyperplasia. Hysteroscopy missed 3 cases of hyperplasia and misdiagnosed one as a polyp and when endometrial hyperplasia was accompanied by polyp hysteroscopic diagnosis was polyp in 6 cases and missed both lesions in one case. As 2D TVS, hysteroscopy did not diagnosed both in one case. When endometrial hyperplasia was accompanied by adenomyosis hysteroscopy diagnosis was hyperplasia (2 cases), adenomyosis (1 case) and missed both lesions in 3 cases.

2D TVS was more sensitive and a little more accurate than hysteroscopy in the diagnosis of endometrial hyperplasia while hysteroscopy was more specific.

For diagnosing endometrial polyp 2D TVS showed sensitivity $76.92 \%$, specificity $91.89 \%$, PPV 76.92\%, NPV $91.89 \%$ and accuracy $88 \%$.

Hysteroscopy showed sensitivity $92.3 \%$, specificity $94.59 \%$, PPV $85.71 \%$, NPV $97.22 \%$ and accuracy $94 \%$ in detecting endometrial polyp.

Hysteroscopy showed highest accuracy for the diagnosis of endometrial polyp and was more sensitive, specific and accurate than 2D TVS in this diagnosis.

$>$ For diagnosing adenomyosis 2D TVS showed sensitivity $68.18 \%$, specificity
98.78\%, PPV 93.75\%, NPV 92.04\% and accuracy $94 \%$

Hysteroscopy showed sensitivity $40.9 \%$, specificity $98.78 \%$, PPV 90\%, NPV $86.17 \%$ and accuracy $88 \%$ in detecting adenomyosis.

2D TVS was more sensitive and accurate than hysteroscopy in the diagnosis of adenomyosis but both show same specificity.

$>$ For differentiating normal from abnormal endometrial cavity_2D TVS show sensitivity $92.3 \%$, specificity $72.72 \%$, PPV $92.3 \%$, NPV $72.72 \%$ and accuracy $88 \%$.these values were obtained with an endometrial thickness of $8 \mathrm{~mm}$.

Hysteroscopy showed sensitivity $78.75 \%$, specificity $95.83 \%$, PPV $98.43 \%$, NPV $57.5 \%$ and accuracy $84 \%$ in detecting endometrial cavitary lesions. For differentiating normal from abnormal endometrial cavity both 2D TVS and hysteroscopy show high accuracy but U/S was more sensitive and a little more accurate than hysteroscopy while the last was more specific. Wang and Mechcatie ${ }^{(16)}$ used hysteroscopy to evaluate 56 women with menorrhagia found that the sensitivity of using the cratered appearance on hysteroscopy to diagnose adenomyosis was about $75 \%$. Wang and Mechcatie (16) described it as good and comparable to that of ultrasound The specificity was $42 \%$, the PPV was $42 \%$ and the NPV was $75 \%$. In our study hysteroscopy was poorly sensitive but highly specific and accurate for adenomyosis. To evaluate the accuracy of TVS in the diagnosis of adenomyosis Bazot and Daraï ${ }^{(17)}$ studied TVS findings in 129 women scheduled for hysterectomy and found that the sensitivity, specificity, and positive and negative predictive values of TVS, $100 \%$ and $83.3 \%$, and $40 \%$ and $82.9 \%$, respectively and the accuracy of TVS was $91.3 \%$. Our data were consistent with these findings. Pennant $\boldsymbol{e t}$ al. (18) measured the endometrial thickness using TVS in 111 perimenopausal women with noncyclic AUB before D\&C An endometrial thickness of $8 \mathrm{~mm}$ showed optimal sensitivity and specificity $(83.9 \%$ and $58.8 \%$, respectively) and $90.4 \%$ NPV for an abnormal endometrium. He concluded that endometrial thickness of $8 \mathrm{~mm}$ or less is less likely to be associated with pathologies in perimenopausal uterine bleeding. The study of Kotdawala et al. (19) was undertaken to investigate cut-off value 
of the endometrial thickness by TVS. This study included 144 perimenopausal women with abnormal bleeding. Their endometrial thickness was measured by TVS and then EMB was performed and the results were correlated with each other. An optimal sensitivity and specificity (83.6 and $56.4 \%$, respectively) and NPV with $95.6 \%$ for detection of abnormal endometrium were obtained with an endometrial thickness of $8 \mathrm{~mm}$. they concluded that an endometrial thickness $>8 \mathrm{~mm}$ is more likely than that of $8 \mathrm{~mm}$ or less to be indicated with EMB in perimenopausal uterine bleeding. To combine findings of normal mid-line echoes from TVS with cut-off levels for endometrial thickness, in order to examine the associated risk of abnormalities in the uterine cavity. Özdemir $\boldsymbol{e t}$ al. (20) compared TVS findings in 355 premenopausal patients complaining AUB with findings obtained from the endometrial sampling which were used as the true values; the found that using TVS, low levels of endometrial thickness ( $\leq$ $7 \mathrm{~mm}$ ) reduced the possibility of abnormalities such as polyps and hyperplasia, but did not exclude them.

Similar findings were obtained by our study, 2D TVS perform well and show high accuracy using $8 \mathrm{~mm}$ as a cut off value; however our sample size was too small to conclude cut- off values for recommendation of screening and endometrial sampling. Kumari and Gupta ${ }^{(15)}$ in their study, 105 patients complaining of AUB were initially evaluated on the same day with both TVS and hysteroscopy. hysteroscopy with biopsy $(n=35)$, curettage $(n=60)$ or hysterectomy $(n=10)$ was performed and the results of TVS and hysteroscopy examination were correlated with the pathological findings. The sensitivity and specificity were $79.0 \%$ and $45.8 \%$ for TVS, and $95.1 \%$ and $83.3 \%$ for hysteroscopy, respectively. The positive and negative predictive values were $83.0 \%$ and $39.3 \%$ for TVS, and $95.1 \%$ and $83.3 \%$ for hysteroscopy, respectively. Eight cases showed a discrepancy between hysteroscopy and the pathologic diagnosis. Kumari and Gupta ${ }^{(15)}$ concluded that hysteroscopy can be better used than TVS in evaluating those patients with AUB. Our study, in contrast, we found that TVS was a little more accurate than hysteroscopy for differentiating normal from abnormal endometrial cavity.

CONCLUSION

From the previous results we concluded that:
- Endometrial hyperplasia was the commonest observed endometrial abnormality in our patient sample and the second most common pathology was the endometrial polyp.

- Transvaginal ultrasound was an excellent tool for investigating uterine cavity abnormality in addition to its known advantages of being noninvasive, safe and relatively inexpensive method for direct assessment of endometrial thickness TVS was a sensitive, specific and accurate method to evaluate the endometrial cavitary lesions. Moreover in differentiating normal fom diseased uterine cavity, in diagnosing endometrial hyperplasia and in diagnosing adenomyosis TVS was more sensitive and accurate than hysteroscopy.

- Hysteroscopy which was known as a safe, popular, and acceptable method of directly visualizing and assessing the uterine cavity, offers an accurate and highly specific method for diagnosing it's abnormality and excellent guide for localization of the pathological lesion before endometrial curettage especially for endometrial polyp. Moreover having accuracy value for detection of endometrial hyperplasia comparable to that of ultrasound is added to its known value as a 'gold standard' in uterine cavity evaluation. However, histopathological diagnosis is always needed to confirm the diagnoses.

- Both 2D TVS and hysteroscopy ignore the diagnoses of one lesion when endometrial hyperplasia, endometrial polyp or adenomyos was found in one case.

\section{REFERENCES}

1. O'Connor VM (2003): Heavy menstrual loss. Part 1: is it really heavy loss? Medicine Today, 4: 51-59.

2. Palep-Singh $M$ and Prentice $A$ (2007): Epidemiology of abnormal uterine bleeding. Clin. Obstet. Gynaecol., 21: 887-890.

3. Speroff L and Fritz MA (2005): The uterus, reproductive physiology.In; Clinical Gynecologic Endocrinology and infertility. $7^{\text {th }}$ ed.: Lippincott Williams and 
Wilkins. Philadelphia, London ,p p:116124.

4. Farquhar C, Ekeroma A, Fentiman G et al. (2001): An evidence based guideline for the management of uterine fibroids. The Australian and New Zealand Journal of Obstetrics and Gynaecology, 41: 125140.

5. Reid RL and Lee JY (1995): Medical management of menorrhagia. Informed, 1: 6-7.

6. Lefebvre G, Vilos G, Allaire $\mathrm{C}$ et al. (2003): The management of uterine leiomyomas. American Journal of Obstetrics and Gynecology, 128: 1-10.

7. Telner DE and Jakubovicz D (2007): Approach to diagnosis and management. Canadian Family Physician, 53: 58-64.

8. Vilos GA, Lefebvre $G$ and Graves GR (2001): Guidelines for the management of abnormal uterine bleeding. J. Obstetrics and Gynaecology Canada, 23: 704-713.

9. Komodiki C (2006): Cervical and corpus uterine cancer. In: Cancer Incidence in Four Member Countries (Cyprus, Egypt, Israel and Jordan) of the Middle East. Freedman LS, Edwards B.K., Ries LAG., Young J.L. USA.,pp: 83-90.

10. Clark TJ (2004): Outpatient hysteroscopy and ultrasonography in the management of endometrial disease. Current Opinion in Obstetrics and Gynecology, 16: 305-311.

11. Oehler MK and Rees MC (2004): Menorrhagia: an update. Acta Obstetricia et Gynecologica Scandinavica, 82: 405422.

12. Derzko CM (1997): Perimenopausal dysfunctional uterine bleeding: physiology and management. Society of Obstetricians and Gynaecologists of Canada, 2:216-235.

13. Brand A, Dubuc-Lissoir J, Ehlen Y et al. (2000): Diagnosis of endometrial cancer in women with abnormal vaginal bleeding. Society of Obstetricians and Gynaecologists of Canada Clinical Practice Guidelines, 8: 1-3.

14. Kumar A and Pearl M (2014): Minilaparotomy versus laparoscopy for gynecologic conditions. Journal of Minimally Invasive Gynecology, 21(1): 109-114.
15. Kumari M and Gupta AS (2017): A prospective comparative study to evaluate the efficacy of ultrasonography and hysteroscopy and their correlation with the histopathology of endometrium in a case of abnormal uterine bleeding. International Journal of Reproduction, Contraception, Obstetrics and Gynecology, 4(6): 1926-35.

16. Wang $A$ and Mechcatie $E$ (2012): Hysteroscopic findings clue adenomyosis diagnosis. Ob. Gyn. News, 36: 6-2.

17. Bazot $M$ and Daraï E (2018): Role of transvaginal sonography and magnetic resonance imaging in the diagnosis of uterine adenomyosis. Fertility and Sterility, 109(3): 389-397.

18. Pennant ME, Mehta $R$, Moody $P$ et al. (2017): Premenopausal abnormal uterine bleeding and risk of endometrial cancer. International Journal of Obstetrics and Gynaecology, 124(3): 404-411.

19. Kotdawala $P$, Kotdawala $S$ and Nagar $N$ (2013): Evaluation of endometrium in peri-menopausal abnormal uterine bleeding. Journal of Mid-Life Health, 4(1): 16-22.

20. Özdemir S, Çelik Ç, Gezginç K et al. (2010): Evaluation of endometrial thickness with transvaginal ultrasonography and histopathology in premenopausal women with abnormal vaginal bleeding. Archives of Gynecology and Obstetrics, 282(4): 395-414. 